This is a post-peer-review, pre-copyedit version of an article published in Journal of Autism and Developmental Disorders. The final authenticated version is available online at:

\title{
Reduced pseudoneglect for physical space, but not mental representations of space, for adults with autistic traits.
}

\author{
Michael C.W. English, Murray T. Maybery, Troy A.W. Visser
}

\section{Abstract}

Neurotypical individuals display a leftward attentional bias, called pseudoneglect, for physical space (e.g. landmark task) and mental representations of space (e.g. mental number line bisection). However, pseudoneglect is reduced in autistic individuals viewing faces, and neurotypical individuals with autistic traits viewing 'greyscale' stimuli, suggesting attention is atypically lateralized in autism. We investigated whether representational pseudoneglect for individuals with autistic traits is also reduced by comparing biases on greyscales, landmark, and mental number line tasks. We found that pseudoneglect was intact only on the representational measure (i.e. the mental number line task), suggesting that mechanisms for atypical lateralization of attention in individuals with autistic traits are specific to processing of physical, visual stimuli.

\section{Introduction}

Asymmetries in brain hemispheric activation have been shown to have contralateral effects on visual attention. Stroke patients with right hemisphere $(\mathrm{RH})$ lesions, for example, focus on stimuli presented primarily in the right visual field (RVF) and ignore (neglect) stimuli presented in the left (Adair \& Barrett, 2008; Bartolomeo, 2007; Heilman, Watson, \& Valenstein, 2003). When completing line bisection tasks (which require indicating the centre of a line) or landmark tasks (which require indicating whether a pre-bisected line has been divided to the left or right of centre), these patients reliably respond as if the centre of the line was towards the right of the true centre.

Interesting, while neglect of the left visual field (LVF) is a common outcome following RH damage, neglect of the RVF following left hemisphere (LH) damage, is relatively less common (Stone, Halligan, \& Greenwood, 1993). Increased connectivity within the RH, as well as inter-hemispheric connectivity that favours information transfer in a right-to-left direction (Siman-Tov et al., 2007), are factors that underlie the widely accepted theory that the RH controls spatial attention for both LVF and RVF, while the LH controls spatial attention only for the RVF (Mesulam, 1981) explaining why neglect of the RVF following LH damage is relatively rare, whereas neglect of the LVF following RH damage is common.

The relatively greater lateralization of spatial attention to the RH (Heilman, 1995; Hellige, 1993) can also be used to account for the fact that healthy individuals show a leftward attentional bias (pseudoneglect; Bowers \& Heilman, 1980). A side effect of the RH lateralization for spatial attention is that stimulus properties in the 'dominant' LVF are exaggerated relative to stimulus properties presented in the other visual field. As a result, neurotypical individuals bisect lines slightly left of the line's true centre (Jewell \& McCourt, 2000) and show leftward attentional biases when making stimulus judgements on the basis of brightness and numerosity (Nicholls, Bradshaw, \& Mattingley, 1999). 
Atypical lateralization of spatial attention is not unique to neglect patients with $\mathrm{RH}$ lesions. It has also been linked to autism spectrum conditions (ASC), neuro-developmental conditions primarily characterized by deficits in social communication and restricted and repetitive behavioural patterns (American Psychiatric Association, 2013). Relative to neurotypical participants, ASC adults usually show a reduced LVF bias in target detection tasks (Wainwright \& Bryson, 1996) and chimeric face identification (Ashwin, Wheelwright, \& Baron-Cohen, 2005), as well as reduced fixation time to the left side of centrally presented faces (Dundas, Best, Minshew, \& Strauss, 2012). Recently, we also reported that neurotypical individuals with relatively high scores on the Autism Spectrum Quotient (AQ; a measure of autistic-like traits in neurotypical participants; Baron-Cohen, Wheelwright, Skinner, Martin, \& Clubley, 2001) show reduced pseudoneglect on the greyscales task (see a description and example stimuli below) compared to participants with lower AQ scores (English, Maybery, \& Visser, 2015). This was the first study to suggest that attenuations of attentional bias found in ASC are also present in a high autistic-trait subset of the neurotypical population and adds to the growing literature indicating that attentional characteristics associated with autism spectrum conditions also manifest to a lesser degree in individuals with higher scores on measures of autisticlike traits (Bayliss \& Kritikos, 2011; Grinter, Van Beek, Maybery, \& Badcock, 2009; Grinter, Maybery, et al., 2009; Rhodes, Jeffery, Taylor, \& Ewing, 2013; Russell-Smith, Maybery, Bayliss, \& Sng, 2012; Sutherland \& Crewther, 2010; for a meta-analysis, see Cribb, Olaithe, Di Lorenzo, Dunlop, \& Maybery, 2016).

The present work examines whether spatially-organized mental representations, like the mental number line, are also altered in individuals with high levels of autistic-like traits. Figure 1 is a conceptual illustration of such a number line, which is described as a series of numbers located on a horizontal azimuth numerically ascending left-to-right (Brooks, Sala, \& Darling, 2014). Past research has shown that when patients with neglect are asked to make a judgement regarding numerical distance, their responses are indicative of a rightward bias on the number line (e.g. selecting ' 7 ' as the midpoint between ' $1-9$ '), with the size of the bias corresponding with neglect severity (Hoeckner et al., 2008; Loftus, Nicholls, Mattingley, Chapman, \& Bradshaw, 2009; Zorzi, Priftis, \& Umiltà, 2002). Conversely, neurotypical participants show a small bias in favour of a lower, more 'leftward' number (Göbel, Calabria, Farnè, \& Rossetti, 2006; Loftus et al., 2009; Longo \& Lourenco, 2007; Nicholls \& Mcllroy, 2010). This representational pseudoneglect is hypothesised to occur due to exaggeration of the distance between numbers that are more "leftward" on the number line (similar to the exaggeration of leftward stimulus features of visual stimuli that results in pseudoneglect), shifting perceptions for the relative location of the central point (Loftus et al., 2009).

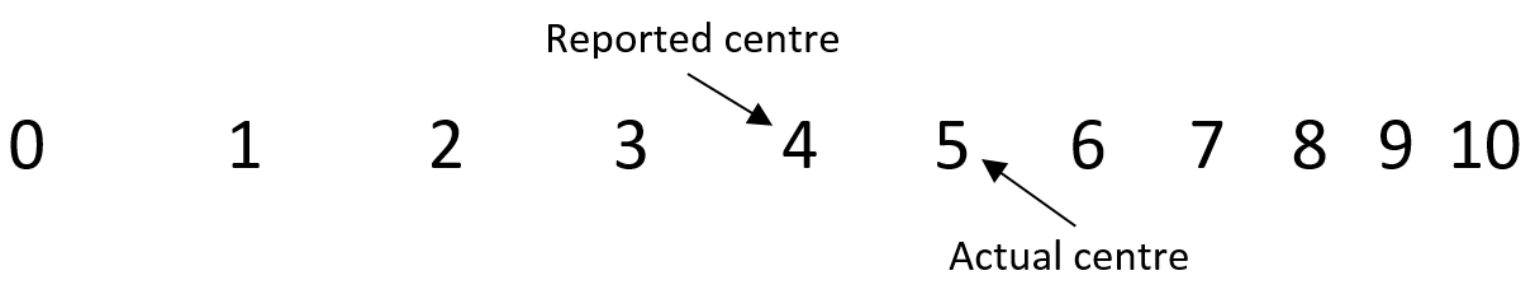

Figure 1. A simplified demonstration of how leftward representational bias occurs in the mental number line. It is suggested that individuals exaggerate the distance between numbers that are further "left" on the mental number line. Thus, when an individual is asked to identify the midpoint of the range $0-10$, 'four' is erroneously reported more often than the correct answer of 'five'.

While both visual and representational stimuli yield leftward biases in neurotypical participants, it is unclear whether both types of bias would also disappear for individuals high in autistic traits. This is partly because evidence from past studies is equivocal with respect to the question of whether these two types of leftward bias have a common neural substrate. One candidate region for such a substrate is the posterior parietal cortex (PPC) due to its involvement in 
many aspects of visual attention, including visual working memory (Berryhill \& Olson, 2008), sustained attention (Husain \& Nachev, 2007; Malhotra, Coulthard, \& Husain, 2009), visually guided reaching (Clower et al., 1996), initiating visually guided saccades (Luna et al., 1998), and activating mentalised spatial maps (Maguire et al., 1998). Non-invasive stimulation of this region has been effective at modulating spatial biases. For example, applying transcranial magnetic stimulation (TMS) to the right PPC of healthy individuals temporarily disrupts its activation (Fierro, Brighina, Piazza, Oliveri, \& Bisiach, 2001), producing a rightward shift in the perceived midpoint of horizontal lines similar to that shown by individuals with RH damage (Bjoertomt, Cowey, \& Walsh, 2002). Likewise, applying TMS over the same region in healthy individuals produces a rightward shift for representational space in the mental number line task, similar to those observed in individuals with right parietal damage (Göbel et al., 2006). Additionally, Loftus and Nicholls (2012) found that pseudoneglect, measured with the luminance-judgement greyscales task (Nicholls et al., 1999), could be eliminated following anodal transcranial direct current stimulation (tDCS) over the left parietal cortex. Anodal tDCS is thought to increase neural excitation of the stimulated site and, in line with Siman-Tov et al.'s (2007) activation-orientation model of attention, likely caused a relative increase in the excitation of neurons in the left parietal cortex which rebalanced the asymmetry in activation between the left and right hemispheres that drives pseudoneglect.

While such studies provide evidence in favour of the notion that PPC activity underlies both physical and representational pseudoneglect, and while several studies have found evidence for both physical and representational biases in the same individuals (Aiello et al., 2012; Longo, Trippier, Vagnoni, \& Lourenco, 2015; Rotondaro, Merola, Aiello, Pinto, \& Doricchi, 2015; Vuilleumier, Ortigue, \& Brugger, 2004; Zorzi, Priftis, Meneghello, Marenzi, \& Umiltà, 2006; Zorzi et al., 2002), other researchers have found that neglect patients who show rightward deviations on physical spatial tasks do not necessarily have similar biases in the representational medium, suggesting that at least partially disparate underlying networks are at play (Aiello et al., 2012; Doricchi, Guariglia, Gasparini, \& Tomaiuolo, 2005; Loetscher \& Brugger, 2009; Loetscher, Nicholls, Towse, Bradshaw, \& Brugger, 2010; Pia et al., 2012; Rossetti et al., 2011; Storer \& Demeyere, 2014; van Dijck, Gevers, Lafosse, \& Fias, 2012). Moreover, there are many fundamental differences between physical and representational stimuli that might result in the recruitment of different neural mechanisms during processing. For one, physical representations, like those in the line bisection and greyscale tasks, act as visual cues that directly influence attentional processes, whereas few or no such cues are present in the representational mental number line. Second, the differences could reflect visual complexity of the task, with complexity generally greater in the visual than representational tasks. Finally, it is possible that task relevant information, such as letter or number identity in representational tasks, may recruit disparate brain areas such as those responsible for memory and language that are unlikely to play a role in processing abstract visual stimuli such as a line or greyscales stimulus.

The results of this study are therefore important for two reasons. First, they address the issue of whether pseudoneglect for mental representations of space is reduced in individuals with high levels of autistic-traits in line with the previously reported reduced pseudoneglect for physical space (English et al., 2015). Second, they provide new evidence concerning the question of whether common or disparate mechanisms underlie physical and representational pseudoneglect. To whit, if variations in levels of autistic-like traits produce similar variations in physical and representational pseudoneglect, this would provide suggestive evidence for common neural mechanisms. On the other hand, if pseudoneglect is not reduced for mental representations of space in individuals with high autistic-trait levels, it would suggest different mechanisms underlie spatial biases in physical and mental representations.

To address these questions, in the present work, we recruited two groups of neurotypical individuals with scores in the upper or lower third of the $A Q$ distribution and measured their response biases on three tasks: greyscales and landmark tasks to observe levels of physical pseudoneglect, and a number line bisection task to observe levels of representational 
pseudoneglect. In line with our earlier findings, we expected reduced levels of physical pseudoneglect in our High AQ group compared to our Low AQ group (English et al., 2015) on both the greyscales and landmark tasks. This would be a replication and a critical extension of this earlier result to a novel task (landmark). If representational pseudoneglect is also attenuated in the group with higher $\mathrm{AQ}$ scores, this would provide the first evidence that autistic traits modulate not only physical but also representational pseudoneglect. This result would also provide evidence for a shared mechanism responsible for attenuation of both forms of pseudoneglect in High AQ (and possibly ASC) individuals.

\section{Methods}

\section{Participants}

Participants were 104 right-handed students recruited at the University of Western

Australia. Students with AQ scores in the upper or lower third of a cohort that had completed the AQ in a previous screening procedure were invited to participate. Fifty-two participants were in each of the Low and High AQ groups.

\section{Materials}

All tasks and questionnaires were presented using Presentation software (Version 17.0, Neurobehavioral Systems) running on HP EliteOne 800 machines using 23" LCD monitors. Participants were seated approximately $50 \mathrm{~cm}$ from the display.

\section{Greyscales Task}

Stimuli for this task were based on those previously used by English et al. (2015). Each trial consisted of two horizontal bars presented above and below the centre of the display. The shading on each bar changed from white to black incrementally along the horizontal azimuth, with one bar effectively being a reversal of the other (see Figure 2). The orientations of the bars were randomized such that the bar that was shaded from the left, white-to-black, was presented equally often as the top or bottom positioned bar. Finally, one bar from each pair was shaded 'darker' than the other bar in the pair. This was achieved by randomly replacing 100 white pixels with black pixels on one bar, and conversely randomly replacing 100 black pixels with white pixels on the other bar, creating a 200-pixel difference in shading between the two bars.

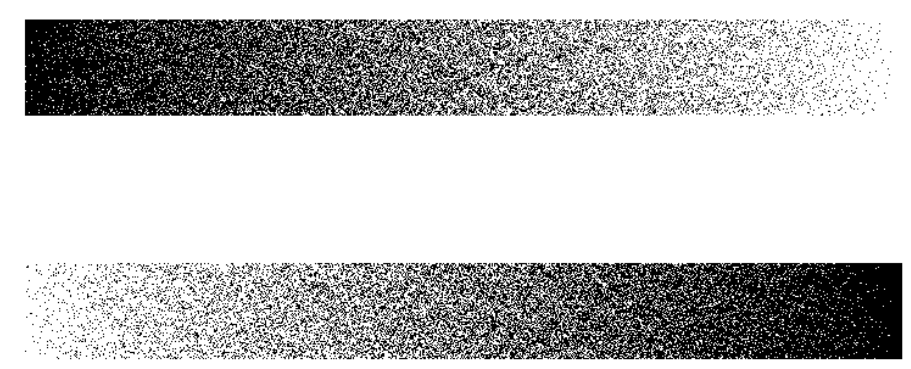

Figure 2. An example of a stimulus presented in the greyscales task.

Each trial began with a fixation cross that was displayed for $1500 \mathrm{~ms}$ before the greyscales stimuli were presented. Participants were instructed to observe the two bars and determine which of the two was 'darker' overall. Participants made their responses using the [T] and [B] keys on a standard keyboard, which indicated the "top" or "bottom" bar respectively. Participants had 5s from the onset of the greyscales stimuli to respond before the trial automatically ended. The task consisted of 120 trials, with factorial combinations of stimulus characteristics such as length and orientation counterbalanced across trials. 


\section{Landmark Task}

Stimuli for this task consisted of a white horizontal bar presented in the centre of the display on a black background. The bar was 481 pixels (px) long and 10px wide and was bisected by a thin $1 \mathrm{px}$ wide, black vertical line. The position of the black line was randomized across trials, appearing in the true centre or $10 p x, 5 p x, 3 p x, 2 p x$, or $1 p x$ to the left or right of centre. A mask consisting of randomly placed black and white pixels (white noise) covered the screen region occupied by the horizontal bar at the beginning of each trial for $1 \mathrm{~s}$ to prevent participants from using positions on the previous trial when making judgements on the current trial.

Each trial began with a mask that was presented for $1000 \mathrm{~ms}$ before the landmark stimuli appeared. Participants were required to decide whether the black vertical line on the white bar was closer to the left or right-hand side of the bar. Participants responded using the [Z] and [/] keyboard keys to indicate that they perceived the bisection to be closer towards the left or right-side of the bar respectively. Participants had $5 \mathrm{~s}$ from the onset of the landmark stimuli within which to respond before the trial automatically ended. The task consisted of 132 trials, with the bisection appearing at all 11 locations an equal number of times.

\section{Number Line Task}

Stimuli for this task were adapted from those used by Loftus et al. (2009). Each trial consisted of a triplet of two-digit numbers, arranged with a central red number and two yellow flankers. There were four sets of flankers (19_55, 53_99, 42_98 and 17_83). For each set, the red central number was always shifted 1, 2 or 5 greater or less than the actual midpoint between the two flankers (e.g. for the flanker pair '19_55', the midpoint is 37 and the possible central numbers included 32, 35, 36, 38, 39 and 42). Whereas the Loftus et al. study presented all numbers on the same horizontal plane, we used a diagonal configuration so that participants made top/bottom judgements instead of left/right judgements (see Figure 3). This change was made to dissociate participants' potential bias towards making left/right key presses from left/right spatial judgements. Number triplets varied factorially with respect to the spatial configuration (spatially ascending/descending (SA/SD), left-to-right) and direction of numerical sequence (numerically ascending/descending (NA/ND), left-to-right). This resulted in four different possible spatial configurations for each triplet configuration (SA-NA, SA-ND, SD-NA, and SD-ND), four sets of flankers, and six possible values for the central number, yielding a total of 96 unique trials presented without repetition.

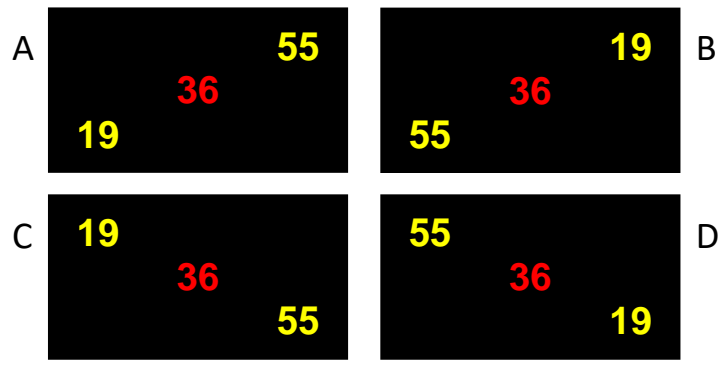

Figure 3. Examples of the four spatial configurations, with each configuration containing one, red central number and two, yellow flanker numbers. Left-to-right; A) spatially ascending, numerically ascending, B) spatially ascending, numerically descending, C) spatially descending, numerically ascending, D) spatially descending, numerically descending.

Each trial began with a centrally presented fixation cross which was displayed for $1000 \mathrm{~ms}$, after which a number triplet was presented. Participants were required to determine if the top flanker or bottom flanker in a triplet was closer in numerical position to the red central number. Participants were instructed to not use arithmetic when making their judgements. Triplets were presented for $3 \mathrm{~s}$, after which the triplet disappeared and participants had $2 \mathrm{~s}$ to make a response 
using the $[\mathrm{T}]$ and $[\mathrm{B}]$ keys to indicate "top" and "bottom" respectively. If a participant did not make a response within $2 \mathrm{~s}$, the trial automatically ended.

\section{Questionnaires}

Levels of autistic-like traits were measured using the Autism Spectrum Quotient (BaronCohen et al., 2001), a 50-item self-report questionnaire that assesses autistic-like traits and behaviours in neurotypical individuals. Items were scored 1-4 using Austin's (2005) method; higher scores represent more autistic-like traits. This scoring method was used rather than the 0-1 method reported by Baron-Cohen et al. (2001) to take advantage of the range of potentially useful information in each item and increase the variability of total AQ scores. Handedness was assessed using the Edinburgh Handedness Inventory (Oldfield, 1971).

\section{Procedure}

Testing took place in a single session that typically lasted 45-60 minutes. At the beginning of the testing session, participants were given a verbal overview of the testing procedure, including the general nature of the tasks and questionnaire involved. Participants then completed the three computer tasks in counterbalanced order across participants. Task-specific instructions were presented to participants on the computer immediately prior to commencing the relevant task. Participants also had the opportunity to complete a number of practice trials at the beginning of each task and take short breaks between tasks.

\section{Results}

Descriptive statistics for the High and Low AQ groups are presented in Table 1. Participant performance was screened for outliers on each of the tasks; if a participant was deemed an outlier for a particular task, their data were excluded from analysis only on that task. No participant was identified as an outlier on more than one task. We conducted one-tail t-tests on the differences in bias between $A Q$ groups because we predicted a reduced left bias in our High $A Q$ group given prior results (English et al., 2015).

\section{Table 1.}

Characteristics of the Low $A Q$ and High AQ comparison groups (standard deviation in parentheses).

\begin{tabular}{lll}
\hline & Low AQ $(n=52,5$ male) & High AQ $(n=52,18$ male $)$ \\
\hline Mean Age (years) & $20.63(4.25)$ & $20.06(3.39)$ \\
Mean AQ & $89.60(7.15)$ & $120.04(6.31)$ \\
\hline
\end{tabular}

The two groups did not differ in mean age $(p=0.45, r=0.07)$, but the Low AQ group contained fewer male participants than the High AQ group $\left[\chi^{2}(1)=9.43, p<0.01\right]$, and so we conducted preliminary versions of the analyses reported below with sex as an additional betweensubjects variable to $A Q$ group. No main effects of sex or interactions between sex and $A Q$ group were reported on any of the task bias measures (all $p^{\prime} s>0.13$, all $\eta_{p}{ }^{2}<0.02$ ). Given this result, we chose to conduct the following analyses without sex entered as a factor in order to focus on effects related to $A Q$ group.

\section{Data Screening}

Several criteria were implemented to ensure that the participants were appropriately engaged with the tasks. Participants were excluded from the analysis of a single task if: A) more than a third of responses were faster than $200 \mathrm{~ms}$ (indicating the participant was anticipating target onset), B) the proportion of top/bottom key presses exceeded the group mean by more than 2.5 standard deviations for the greyscales or mental number line task or $\mathrm{C}$ ) the proportion of left/right key presses for the two most extreme (and easiest) bisection locations exceeded the group mean by more than 2.5 standard deviations for the landmark task. One participant met criteria A (landmark 
task, High $A Q$ ), five participants met criteria $B$ (greyscales task, two Low $A Q$; mental number line task, one Low $A Q$ and two High $A Q$ ) and seven participants met criteria $C$ (three Low $A Q$, four High AQ). No participant met exclusion criteria for multiple tasks. Finally, one participant was excluded from all analyses because her accuracy on multiple tasks was less than $50 \%$ (low $A Q$ ), and one participant was excluded from the analysis for the mental number line task due to a data recording error (Low AQ).

\section{Greyscales Task}

Task accuracy was calculated as the percentage of trials for which a participant correctly identified the darker of the two bars on a given trial. Leftward spatial bias was calculated as the percentage of trials for which a participant selected the bar which had the darker end oriented towards the left-side of the screen (e.g. the top bar in Figure 2), regardless of whether the selected bar was actually darker overall.

Accuracy for both the Low $A Q(M=56.23 \%, S D=6.17 \%)$ and High $A Q$ group $(M=57.38 \%$. $\mathrm{SD}=6.66 \%$ ) was above chance level (largest $p<0.001$, smallest $r=0.71$ ), with no difference between the groups ( $p=0.37, r=0.09$ ). Pseudoneglect (leftward spatial bias) differed significantly from chance levels for the Low AQ group ( $\mathrm{M}=60.38 \%, \mathrm{SD}=18.23 \% ; p<0.001, r=0.50)$, but only marginally so for the High AQ group ( $\mathrm{M}=54.54 \%, \mathrm{SD}=16.32 \% ; p=0.05, r=0.27$ ). Additionally, pseudoneglect was significantly higher in the Low $\mathrm{AQ}$ group relative to the High AQ group, $t(99)=1.70, p=0.046, r$ $=0.17$. Taken together, these results show an even stronger pattern than in our previous work (English et al., 2015), with only marginal levels of pseudoneglect seen in the High AQ group and a larger difference in pseudoneglect between $A Q$ groups ( $r=0.17$ vs. $r=0.12$ in our previous study). Greater levels of autistic traits in the High AQ group of the present study relative to the previous study might account for this stronger pattern (High AQ: $M=120.04$ vs $M=115.14$; see Cribb et al. (2016) for simulations of the influence of $A Q$ group separation).

\section{Landmark Task}

Task accuracy was calculated as the percentage of trials on which participants correctly identified the location of the bisection towards the left or right side of the horizontal bar, excluding trials where the bisection was presented at centre. Leftward spatial bias was calculated as the overall percentage of trials in which participants indicated that the bisection was closer to the rightside of the bar (as increased rightward responses suggest a relative perceptual exaggeration of the left side of space).

Accuracy for both the Low $A Q(M=69.43 \%, S D=8.27 \%)$ and High $A Q$ group ( $M=68.48 \%$, $\mathrm{SD}=8.64 \%$ ) was above chance level (largest $p<0.001$, smallest $r=0.91$ ), with no difference between the groups $(p=0.59, r=0.06)$. Pseudoneglect differed significantly from chance levels in the Low AQ group ( $\mathrm{M}=55.89 \%, \mathrm{SD}=15.72 \% ; p=0.01, r=0.35$ ), but not in the High $\mathrm{AQ}$ group ( $\mathrm{M}=50.57 \%$, $\mathrm{SD}=15.15 \% ; p=0.51, r=0.04)$. Additionally, pseudoneglect was significantly higher in the Low $\mathrm{AQ}$ group than in the High AQ group, $t(93)=1.68, p=0.048, r=0.17$. Taken together, these findings suggest the Low $A Q$ group showed pseudoneglect while the High AQ group did not, conceptually replicating findings using the greyscales task here and in our previous study (English et al., 2015).

\section{Number Line Task}

Response accuracy was measured as the percentage of trials on which participants correctly identified the flanker number that was closest in numerical position to the central number. A summary of the mean accuracy for each of the four configurations (illustrated in Figure 3 ) is provided in Table 2. A repeated measures analysis of variance (ANOVA) with the factors configuration type and $A Q$ group revealed a main effect of configuration type on accuracy, $F(7,96)=$ $7.81, p<0.001, \eta_{p}{ }^{2}=0.08$. However, there was no main effect of AQ group $\left(p=0.60, \eta_{p}^{2}<0.01\right)$, or configuration $\times A Q$ group interaction $\left(p=0.96, \eta_{p}^{2}<0.01\right)$. Accuracy for the Low AQ group 
$(\mathrm{M}=65.48 \%, \mathrm{SD}=7.17 \%)$ and High $\mathrm{AQ}$ group $(\mathrm{M}=64.63 \%, \mathrm{SD}=8.64 \%)$ were both above chance levels (largest $p<0.001$, smallest $r=0.86)$, with no difference between the groups $(p=0.89, r=0.01$ ). 
Table 2.

Number task accuracy across trial configurations (standard deviation in parentheses)

\begin{tabular}{lllll}
\hline & Configuration A & Configuration B & Configuration C & Configuration D \\
\cline { 2 - 5 } Low AQ & $67.35 \%(11.29 \%)$ & $67.09 \%(9.33 \%)$ & $65.22 \%(10.98 \%)$ & $62.22 \%(11.13 \%)$ \\
High AQ & $66.42 \%(11.09 \%)$ & $67.00 \%(12.37 \%)$ & $64.33 \%(9.89 \%)$ & $60.75 \%(13.37 \%)$ \\
\hline
\end{tabular}

Representational pseudoneglect (leftward representational bias) was calculated as the percentage of trials in which participants indicated that the flanker that was of a higher value was closer in numerical position to the central number. In the context of this study, in keeping with convention (Brooks et al., 2014), we refer to the numerically higher number as being positioned "rightward" of the central number, as the mental number line is considered to be a series of numbers that ascend in value from left-to-right. Therefore, an increased percentage of rightward responses would be indicative of exaggeration of the left side of the mental representation of the number line. Note that the words "left" or "right" in this task refer to a number's position on the mental number line, not to its location on the display. To ensure that participants were not preferentially responding to the flanker number that was physically on the left side or right side of the screen, single-sample t-tests were conducted on both $A Q$ groups to determine if responses to items presented on the left side of the screen differed from chance levels. Neither AQ group reported a significant bias towards flankers that were presented on one side of the screen (smallest $p=0.44$, largest $r=0.11$ ).

Representational pseudoneglect for both the Low $A Q$ group $(M=58.17 \%, S D=11.80 \%)$ and High AQ group ( $M=54.53 \%, S D=12.62 \%)$ exceeded chance levels (smallest $p=0.01$, largest $r=0.34$ ). There was no significant difference in the levels of representational pseudoneglect between $A Q$ groups ( $p=0.21, r=0.08)$, which substantially differs from the results reported for the greyscales and landmark tasks, as well as our previous study (English et al., 2015), where physical pseudoneglect was attenuated in High AQ participants.

\section{Discussion}

The aim of this study was to determine if representational pseudoneglect is attenuated in a High AQ sample as has been reported for physical pseudoneglect (English et al., 2015). This was achieved by measuring response biases on tasks that require visual assessment of physical stimuli (the landmark and greyscales tasks), and a task that requires assessment of an internalized representation of space (the mental number line bisection task). If pseudoneglect were reduced for all tasks in the High AQ group relative to the Low AQ group, this would be evidence for a common neural mechanism underlying reduced pseudoneglect for physical and representational measures of space in individuals with high levels of autistic traits. However, if pseudoneglect were only reduced on the physical spatial tasks, and unaltered on the mental number line task, this would instead suggest that an attentional difference is present in High AQ individuals that specifically affects attentional bias in processing of visually-presented stimuli.

Regarding physical pseudoneglect, the study has two key findings. First, it replicates our previous evidence for attenuated pseudoneglect amongst High AQ participants on a greyscales task (English et al., 2015). Second, it extends and generalize our previous findings by showing that physical pseudoneglect on the landmark task is also reduced in a High AQ group. This is particularly important, as it may have been possible that the attenuated pseudoneglect reported in the previous study was specific to the greyscales stimuli. To the contrary, the present results confirm that High $A Q$ participants show a broad reduction in physical pseudoneglect relative to their Low $A Q$ peers. 
The results from the mental number line task answer two key questions about the relationship between $A Q$ and representational pseudoneglect. First, whereas bias scores for the High AQ group did not significantly differ from chance levels on either measure of physical pseudoneglect, this was not the case for the number line task. Here, the High AQ group showed a significant level of representational pseudoneglect. Second, whereas physical pseudoneglect was reduced in the High $A Q$ group relative to the Low $A Q$ group for the landmark and greyscales task, levels of representational pseudoneglect were comparable between $A Q$ groups in the number line task. Taken together, these results suggest high levels of autistic-like traits do not influence spatiallyorganized mental representations, indicating that unique mechanisms underlie physical and representational spatial biases in this group.

Why might pseudoneglect differ between physical and mental spatial representations for High AQ participants? The limited research into asymmetries in spatial attention with respect to ASC restricts our ability to form definite conclusions. However, one likely possibility is that attenuation of physical pseudoneglect arises from processes directly related to the visual processing system that are not involved in the number line task (which does not require judgements based on visual stimulus characteristics). It is well documented that attention-related visual processing in autism is markedly different than in neurotypical individuals. In particular, one of the most widely reported differences is with respect to the global and local processing of visually presented stimuli. Whereas most neurotypical individuals show a preference for processing visual stimuli in a holistic or global manner, ASC and High AQ individuals show a preference for a more piecemeal local processing style (Bayliss \& Kritikos, 2011; Cribb et al., 2016; Grinter, Maybery, et al., 2009; Grinter, Van Beek, et al., 2009; Rhodes et al., 2013; Russell-Smith et al., 2012; Sutherland \& Crewther, 2010). This difference in preferential processing styles is particularly relevant here because, like pseudoneglect, global processing is linked to $\mathrm{RH}$ activation while local processing is associated with $\mathrm{LH}$ activation (Evans, Shedden, Hevenor, \& Hahn, 2000; Flevaris, Bentin, \& Robertson, 2010; Hübner \& Studer, 2009; Lux et al., 2004; Malinowski, Hübner, Keil, \& Gruber, 2002; Volberg \& Hübner, 2004; Weissman \& Woldorff, 2005; Yamaguchi, Yamagata, \& Kobayashi, 2000).

Given this parallel, we tentatively hypothesize that differences in hemispheric activation in High AQ and ASC specifically affect processes related to visual attention and stimulus evaluation. On this notion, in the absence of the need to evaluate a visual stimulus, hemispheric activation biases do not affect performance. While this dissociation might reflect differences unique to High $A Q$ and ASC, it may alternatively reflect similar mechanisms underlying dissociations in $\mathrm{RH}$-damaged patients, where neglect on visual and representational tasks has not always been found to co-occur (Doricchi et al., 2005; Loetscher \& Brugger, 2009; Loetscher et al., 2010; Pia et al., 2012; Rossetti et al., 2011; Storer \& Demeyere, 2014; van Dijck et al., 2012). These dissociations may be driven by the activation of different regions depending on the physical or representational nature of the task. For example, line bisection judgements are associated with activation of the striate, extrastriate visual cortex and parietal lobes, with noted RH lateralization (Doricchi \& Angelelli, 1999; Fink et al., 2000). However, judgements of number positioning are relatively less lateralized to the $\mathrm{RH}$, and are associated with bilateral intraparietal sulcus activation, as well as activation in the left precentral gyrus and prefrontal areas (Dehaene, 2004; Dehaene, Piazza, Pinel, \& Cohen, 2003; Doricchi et al., 2005; Walsh, 2003). If spatial attention shows reduced RH lateralization for individuals with high levels of autistic traits, these regional differences in activation may account for why we found differences in between the two AQ groups on physical but not representational pseudoneglect.

Overall, the absence of reduced representational pseudoneglect for High AQ individuals is evidence against the notion of a common mechanism underlying physical and representational pseudoneglect. What then, may account for the results of previous studies, that found that using TMS or tDCS to alter the activation of the posterior parietal cortex shifted attentional biases for physical and representational measures of space in the contralateral direction of the stimulated hemisphere (Göbel et al., 2006; Göbel, Walsh, \& Rushworth, 2001; Loftus \& Nicholls, 2012)? As 
mentioned earlier, while both task types are associated with parietal activation, several cortical regions are only activated when completing either physical or representational spatial tasks specifically (Dehaene, 2004; Dehaene et al., 2003; Doricchi \& Angelelli, 1999; Doricchi et al., 2005; Fink et al., 2000; Walsh, 2003). A potential explanation that is compatible with the account above is that stimulation of the PPC may lead to attentional changes for both task types, while other key structures are unaffected. This may be of key importance as unaffected structures might compensate for changes in parietal activity. For example, in the absence of external stimulation, relatively intact right prefrontal working memory structures, which are linked to building up the mental number line (Doricchi et al., 2005) may compensate for reduced parietal activation that is related to high levels of autistic traits.

To summarize, the purpose of this study was to determine if previously reported alterations in physical spatial bias found between $A Q$ groups also occurred in spatially-organized mental representations, and to generalize our previous findings (English et al., 2015) using an alternative measure of attentional spatial bias (the landmark task). While we replicated the attenuation of physical pseudoneglect in High AQ participants on both tasks, Low and High AQ groups showed comparable representational pseudoneglect on the mental number line task. We also hypothesize that the PPCs play a specific role in processing physical spatial representations but not in tasks requiring judgements about mental spatial representations, thus potentially explaining task dissociations in our High AQ group. Finally, it should also be noted that although the findings of this study are likely to be indicative of what might be found in a clinical ASD sample, it is important to replicate our findings using such a sample in future work. While research into lateralization of spatial attention in regard to ASD is a relatively novel line of inquiry, atypical asymmetries are relatively simple to observe and, with further understanding of the underlying mechanisms, such measures could be valuable in the assessment and monitoring of neurological functioning in ASD.

\section{References}

Adair, J. C., \& Barrett, A. M. (2008). Spatial neglect: clinical and neuroscience review: a wealth of information on the poverty of spatial attention. Annals of the New York Academy of Sciences, 1142, 21-43. http://doi.org/10.1196/annals.1444.008

Aiello, M., Jacquin-Courtois, S., Merola, S., Ottaviani, T., Tomaiuolo, F., Bueti, D., ... Doricchi, F. (2012). No inherent left and right side in human "mental number line": Evidence from right brain damage. Brain, 135(8), 2492-2505. http://doi.org/10.1093/brain/aws114

American Psychiatric Association. (2013). Diagnostic and statistical manual of mental disorders: DSM-5 (5th ed.). Arlington, VA: American Psychiatric Publishing.

Ashwin, C., Wheelwright, S., \& Baron-Cohen, S. (2005). Laterality Biases to Chimeric Faces in Asperger Syndrome: What is Right About Face-Processing? Journal of Autism and Developmental Disorders, 35(2), 183-196. http://doi.org/10.1007/s10803-004-1997-3

Austin, E. J. (2005). Personality correlates of the broader autism phenotype as assessed by the Autism Spectrum Quotient (AQ). Personality and Individual Differences, 38(2), 451-460. http://doi.org/10.1016/j.paid.2004.04.022

Baron-Cohen, S., Wheelwright, S., Skinner, R., Martin, J., \& Clubley, E. (2001). The Autism-Spectrum Quotient (AQ): Evidence from Asperger Syndrome/High-Functioning Autism, Males and Females, Scientists and Mathematicians. Journal of Autism and Developmental Disorders, 31(1), 5-17. http://doi.org/10.1023/A:1005653411471

Bartolomeo, P. (2007). Visual neglect. Current Opinion in Neurology, 20(4), 381-6. http://doi.org/10.1097/WCO.0b013e32816aa3a3

Bayliss, A. P., \& Kritikos, A. (2011). Brief Report: Perceptual Load and the Autism Spectrum in Typically Developed Individuals. Journal of Autism and Developmental Disorders, 41(11), 1573- 
1578. http://doi.org/10.1007/s10803-010-1159-8

Berryhill, M. E., \& Olson, I. R. (2008). The right parietal lobe is critical for visual working memory. Neuropsychologia, 46(7), 1767-1774. http://doi.org/10.1016/j.neuropsychologia.2008.01.009

Bjoertomt, O., Cowey, A., \& Walsh, V. (2002). Spatial neglect in near and far space investigated by repetitive transcranial magnetic stimulation. Brain, 125(9), 2012-22. http://doi.org/10.1093/brain/awf211

Bowers, D., \& Heilman, K. M. (1980). Pseudoneglect: Effects of hemispace on a tactile line bisection task. Neuropsychologia, 18(4-5), 491-498. http://doi.org/10.1016/0028-3932(80)90151-7

Brooks, J. L., Sala, S. Della, \& Darling, S. (2014). Representational pseudoneglect: A review. Neuropsychology Review, 24, 148-165. http://doi.org/10.1007/s11065-013-9245-2

Clower, D. M., Hoffman, J. M., Votaw, J. R., Faber, T. L., Woods, R. P., \& Alexander, G. E. (1996). Role of posterior parietal cortex in the recalibration of visually guided reaching. Nature, 383(6601), 618-621. http://doi.org/10.1038/383618a0

Cribb, S. J., Olaithe, M., Di Lorenzo, R., Dunlop, P. D., \& Maybery, M. T. (2016). Embedded Figures Test Performance in the Broader Autism Phenotype: A Meta-analysis. Journal of Autism and Developmental Disorders. http://doi.org/10.1007/s10803-016-2832-3

Dehaene, S. (2004). Arithmetic and the brain. Current Opinion in Neurobiology, 14(2), 218-224. http://doi.org/10.1016/j.conb.2004.03.008

Dehaene, S., Piazza, M., Pinel, P., \& Cohen, L. (2003). THREE PARIETAL CIRCUITS FOR NUMBER PROCESSING. Cognitive Neuropsychology, 20(3-6), 487-506. http://doi.org/10.1080/02643290244000239

Doricchi, F., \& Angelelli, P. (1999). Misrepresentation of horizontal space in left unilateral neglect: role of hemianopia. Neurology, 52(March 2015), 1845-1852.

http://doi.org/10.1212/WNL.52.9.1845

Doricchi, F., Guariglia, P., Gasparini, M., \& Tomaiuolo, F. (2005). Dissociation between physical and mental number line bisection in right hemisphere brain damage. Nature Neuroscience, 8(12), 1663-1665. http://doi.org/10.1038/nn1563

Dundas, E. M., Best, C. A., Minshew, N. J., \& Strauss, M. S. (2012). A lack of left visual field bias when individuals with autism process faces. Journal of Autism and Developmental Disorders, 42(6), 1104-11. http://doi.org/10.1007/s10803-011-1354-2

English, M. C. W., Maybery, M. T., \& Visser, T. A. W. (2015). Individuals with Autistic-Like Traits Show Reduced Lateralization on a Greyscales Task. Journal of Autism and Developmental Disorders, 45(10), 3390-3395. http://doi.org/10.1007/s10803-015-2493-7

Evans, M. A., Shedden, J. M., Hevenor, S. J., \& Hahn, M. C. (2000). The effect of variability of unattended information on global and local processing: evidence for lateralization at early stages of processing. Neuropsychologia, 38(3), 225-239. http://doi.org/10.1016/S00283932(99)00080-9

Fierro, B., Brighina, F., Piazza, A., Oliveri, M., \& Bisiach, E. (2001). Timing of right parietal and frontal cortex activity in visuo-spatial perception: a TMS study in normal individuals. Neuroreport, 12(11), 2605-2607. http://doi.org/10.1097/00001756-200108080-00062

Fink, G. R., Marshall, J. C., Shah, N. J., Weiss, P. H., Halligan, P. W., Ziemons, K., ... Freund, H. J. (2000). Line bisection judgments implicate right parietal cortex and cerebellum as assessed by fMRI. Neurology, 54(6), 1324-31. http://doi.org/10.1212/WNL.54.6.1324

Flevaris, A. V, Bentin, S., \& Robertson, L. C. (2010). Local or global? Attentional selection of spatial frequencies binds shapes to hierarchical levels. Psychological Science, 21(3), 424-31.

http://doi.org/10.1177/0956797609359909 
Göbel, S. M., Calabria, M., Farnè, A., \& Rossetti, Y. (2006). Parietal rTMS distorts the mental number line: Simulating "spatial" neglect in healthy subjects. Neuropsychologia, 44(6), 860-868. http://doi.org/10.1016/j.neuropsychologia.2005.09.007

Göbel, S. M., Walsh, V., \& Rushworth, M. F. S. (2001). The mental number line and the human angular gyrus. Neurolmage, 14(6), 1278-89. http://doi.org/10.1006/nimg.2001.0927

Grinter, E. J., Maybery, M. T., Van Beek, P. L., Pellicano, E., Badcock, J. C., \& Badcock, D. R. (2009). Global visual processing and self-rated autistic-like traits. Journal of Autism and Developmental Disorders, 39(9), 1278-90. http://doi.org/10.1007/s10803-009-0740-5

Grinter, E. J., Van Beek, P. L., Maybery, M. T., \& Badcock, D. R. (2009). Brief report: visuospatial analysis and self-rated autistic-like traits. Journal of Autism and Developmental Disorders, 39(4), 670-7. http://doi.org/10.1007/s10803-008-0658-3

Heilman, K. M. (1995). Attentional asymmetries. In R. J. Davidson \& K. Hugdahl (Eds.), Brain Asymmetry (pp. 217-234). Cambridge: MIT Press.

Heilman, K. M., Watson, R. T., \& Valenstein, E. (2003). Neglect and related disorders. In K. M. Heilman \& E. Valenstein (Eds.), Clinical Neuropsychology (pp. 279-336). New York: Oxford University Press.

Hellige, J. B. (1993). Hemispheric Asymmetry: What's Right and What's Left. (S. M. Kosslyn, Ed.). Cambridge: Harvard University Press.

Hoeckner, S. H., Moeller, K., Zauner, H., Wood, G., Haider, C., Gaßner, A., \& Nuerk, H.-C. (2008). Impairments of the mental number line for two-digit numbers in neglect. Cortex, 44(4), 429438. http://doi.org/10.1016/j.cortex.2007.09.001

Hübner, R., \& Studer, T. (2009). Functional hemispheric differences for the categorization of global and local information in naturalistic stimuli. Brain and Cognition, 69(1), 11-18.

http://doi.org/10.1016/j.bandc.2008.04.009

Husain, M., \& Nachev, P. (2007). Space and the parietal cortex. Trends in Cognitive Sciences, 11(1), 30-36. http://doi.org/10.1016/j.tics.2006.10.011

Jewell, G., \& McCourt, M. E. (2000). Pseudoneglect: a review and meta-analysis of performance factors in line bisection tasks. Neuropsychologia, 38(1), 93-110. http://doi.org/10.1016/S00283932(99)00045-7

Loetscher, T., \& Brugger, P. (2009). Random number generation in neglect patients reveals enhanced response stereotypy, but no neglect in number space. Neuropsychologia, 47(1), 276-279. http://doi.org/10.1016/j.neuropsychologia.2008.08.005

Loetscher, T., Nicholls, M. E. R., Towse, J. N., Bradshaw, J. L., \& Brugger, P. (2010). Lucky numbers: Spatial neglect affects physical, but not representational, choices in a Lotto task. Cortex, 46(5), 685-690. http://doi.org/10.1016/j.cortex.2009.06.010

Loftus, A. M., \& Nicholls, M. E. R. (2012). Testing the activation-orientation account of spatial attentional asymmetries using transcranial direct current stimulation. Neuropsychologia, 50(11), 2573-6. http://doi.org/10.1016/j.neuropsychologia.2012.07.003

Loftus, A. M., Nicholls, M. E. R., Mattingley, J. B., Chapman, H. L., \& Bradshaw, J. L. (2009). Pseudoneglect for the bisection of mental number lines. Quarterly Journal of Experimental Psychology, 62(5), 925-945. http://doi.org/10.1080/17470210802305318

Longo, M. R., \& Lourenco, S. F. (2007). Spatial attention and the mental number line: Evidence for characteristic biases and compression. Neuropsychologia, 45(7), 1400-1407. http://doi.org/10.1016/j.neuropsychologia.2006.11.002

Longo, M. R., Trippier, S., Vagnoni, E., \& Lourenco, S. F. (2015). Right hemisphere control of visuospatial attention in near space. Neuropsychologia, 70, 350-357. 
http://doi.org/10.1016/j.neuropsychologia.2014.10.035

Luna, B., Thulborn, K. R., Strojwas, M. H., McCurtain, B. J., Berman, R. A., Genovese, C. R., \& Sweeney, J. A. (1998). Dorsal cortical regions subserving visually guided saccades in humans: An fMRI study. Cerebral Cortex, 8(1), 40-47. http://doi.org/10.1093/cercor/8.1.40

Lux, S., Marshall, J. C., Ritzl, A., Weiss, P. H., Pietrzyk, U., Shah, N. J., ... Fink, G. R. (2004). A functional magnetic resonance imaging study of local/global processing with stimulus presentation in the peripheral visual hemifields. Neuroscience, 124(1), 113-20.

http://doi.org/10.1016/j.neuroscience.2003.10.044

Maguire, E. a, Burgess, N., Donnett, J. G., Frackowiak, R. S., Frith, C. D., \& O'Keefe, J. (1998). Knowing where and getting there: a human navigation network. Science, 280(5365), 921-924. http://doi.org/10.1126/science.280.5365.921

Malhotra, P., Coulthard, E. J., \& Husain, M. (2009). Role of right posterior parietal cortex in maintaining attention to spatial locations over time. Brain, 132(3), 645-660. http://doi.org/10.1093/brain/awn350

Malinowski, P., Hübner, R., Keil, A., \& Gruber, T. (2002). The influence of response competition on cerebral asymmetries for processing hierarchical stimuli revealed by ERP recordings. Experimental Brain Research, 144(1), 136-139. http://doi.org/10.1007/s00221-002-1057-1

Mesulam, M. M. (1981). A cortical network for directed attention and unilateral neglect. Annals of Neurology, 10(4), 309-325. http://doi.org/10.1002/ana.410100402

Nicholls, M. E. R., Bradshaw, J. L., \& Mattingley, J. B. (1999). Free-viewing perceptual asymmetries for the judgement of brightness, numerosity and size. Neuropsychologia, 37(3), 307-314. http://doi.org/10.1016/S0028-3932(98)00074-8

Nicholls, M. E. R., \& Mcllroy, A. M. (2010). Spatial cues affect mental number line bisections. Experimental Psychology, 57(4), 315-9. http://doi.org/10.1027/1618-3169/a000037

Oldfield, R. C. (1971). The assessment and analysis of handedness: The Edinburgh inventory. Neuropsychologia, 9(1), 97-113. http://doi.org/10.1016/0028-3932(71)90067-4

Pia, L., Neppi-Mòdona, M., Cremasco, L., Gindri, P., Dal Monte, O., \& Folegatti, A. (2012). Functional independence between numerical and visual space: Evidence from right brain-damaged patients. Cortex, 48(10), 1351-1358. http://doi.org/10.1016/j.cortex.2012.04.005

Rhodes, G., Jeffery, L., Taylor, L., \& Ewing, L. (2013). Autistic traits are linked to reduced adaptive coding of face identity and selectively poorer face recognition in men but not women. Neuropsychologia, 51(13), 2702-2708. http://doi.org/10.1016/j.neuropsychologia.2013.08.016

Rossetti, Y., Jacquin-Courtois, S., Aiello, M., Ishihara, M., Brozzoli, C., \& Doricchi, F. (2011). Neglect "Around the Clock": Dissociating number and spatial neglect in right brain damage. In S. Dehaene \& E. Brannon (Eds.), Space, Time and Number in the Brain (pp. 149-173). London, United Kingdom: Elsevier. http://doi.org/10.1016/B978-0-12-385948-8.00011-6

Rotondaro, F., Merola, S., Aiello, M., Pinto, M., \& Doricchi, F. (2015). Dissociation between line bisection and mental-number-line bisection in healthy adults. Neuropsychologia, 75, 565-576. http://doi.org/10.1016/j.neuropsychologia.2015.07.016

Russell-Smith, S. N., Maybery, M. T., Bayliss, D. M., \& Sng, A. A. H. (2012). Support for a Link Between the Local Processing Bias and Social Deficits in Autism: An Investigation of Embedded Figures Test Performance in Non-Clinical Individuals. Journal of Autism and Developmental Disorders, 42(11), 2420-2430. http://doi.org/10.1007/s10803-012-1506-z

Siman-Tov, T., Mendelsohn, A., Schonberg, T., Avidan, G., Podlipsky, I., Pessoa, L., ... Hendler, T. (2007). Bihemispheric Leftward Bias in a Visuospatial Attention-Related Network. Journal of Neuroscience, 27(42), 11271-11278. http://doi.org/10.1523/JNEUROSCI.0599-07.2007 
Stone, S. P., Halligan, P. W., \& Greenwood, R. . (1993). The Incidence of Neglect Phenomena and Related Disorders in Patients with an Acute Right or Left Hemisphere Stroke. Age and Ageing, 22(1), 46-52. http://doi.org/10.1093/ageing/22.1.46

Storer, L., \& Demeyere, N. (2014). Disruptions to number bisection after brain injury: Neglecting parts of the mental number line or working memory impairments? Brain and Cognition, 86(1), 116-123. http://doi.org/10.1016/j.bandc.2014.02.004

Sutherland, A., \& Crewther, D. P. (2010). Magnocellular visual evoked potential delay with high autism spectrum quotient yields a neural mechanism for altered perception. Brain, 133(7), 2089-97. http://doi.org/10.1093/brain/awq122

van Dijck, J., Gevers, W., Lafosse, C., \& Fias, W. (2012). The heterogeneous nature of number-space interactions. Frontiers in Human Neuroscience, 5(January), 1-13. http://doi.org/10.3389/fnhum.2011.00182

Volberg, G., \& Hübner, R. (2004). On the role of response conflicts and stimulus position for hemispheric differences in global/local processing: an ERP study. Neuropsychologia, 42(13), 1805-1813. http://doi.org/10.1016/j.neuropsychologia.2004.04.017

Vuilleumier, P., Ortigue, S., \& Brugger, P. (2004). The number space and neglect. Cortex, 40(2), 399410. http://doi.org/10.1016/S0010-9452(08)70134-5

Wainwright, J. A., \& Bryson, S. E. (1996). Visual-spatial orienting in autism. Journal of Autism and Developmental Disorders, 26(4), 423-438. http://doi.org/10.1007/BF02172827

Walsh, V. (2003). A theory of magnitude: Common cortical metrics of time, space and quantity. Trends in Cognitive Sciences, 7(11), 483-488. http://doi.org/10.1016/j.tics.2003.09.002

Weissman, D. H., \& Woldorff, M. G. (2005). Hemispheric Asymmetries for Different Components of Global/Local Attention Occur in Distinct Temporo-parietal Loci. Cerebral Cortex, 15(6), 870876. http://doi.org/10.1093/cercor/bhh187

Yamaguchi, S., Yamagata, S., \& Kobayashi, S. (2000). Cerebral asymmetry of the "top-down" allocation of attention to global and local features. The Journal of Neuroscience : The Official Journal of the Society for Neuroscience, 20(9), RC72.

Zorzi, M., Priftis, K., Meneghello, F., Marenzi, R., \& Umiltà, C. (2006). The spatial representation of numerical and non-numerical sequences: Evidence from neglect. Neuropsychologia, 44(7), 1061-1067. http://doi.org/10.1016/j.neuropsychologia.2005.10.025

Zorzi, M., Priftis, K., \& Umiltà, C. (2002). Brain damage: neglect disrupts the mental number line. Nature, 417(6885), 138-139. http://doi.org/10.1038/417138a 